\title{
NAVIGATION ON A GREAT ELLIPSE
}

\section{Tseng Wei-Kuo}

Department of Merchant Marine, National Taiwan Ocean University, Keelung, Taiwan, R.O.C, wilco0917@yahoo.com.tw

Lee Hsuan-Shih

Department of Shipping and Transportation Management, National Taiwan Ocean University, Keelung, Taiwan, R.O.C.

Follow this and additional works at: https://jmstt.ntou.edu.tw/journal

Part of the Engineering Commons

\section{Recommended Citation}

Wei-Kuo, Tseng and Hsuan-Shih, Lee (2010) "NAVIGATION ON A GREAT ELLIPSE," Journal of Marine Science and Technology. Vol. 18: Iss. 3, Article 6.

DOI: $10.51400 / 2709-6998.1882$

Available at: https://jmstt.ntou.edu.tw/journal/vol18/iss3/6

This Research Article is brought to you for free and open access by Journal of Marine Science and Technology. It has been accepted for inclusion in Journal of Marine Science and Technology by an authorized editor of Journal of Marine Science and Technology. 


\title{
NAVIGATION ON A GREAT ELLIPSE
}

\author{
Wei-Kuo Tseng* and Hsuan-Shih Lee**
}

Key words: great circle, great ellipse, geodesics.

\begin{abstract}
This paper presents contact formulae for the computation of the position, the distance, and the azimuth along a great ellipse. The proposed alternative formulae are to be primarily used for accurate sailing calculations on the ellipsoid in a GIS environment as in ECDIS and other ECS. Among the ECDIS Requirements is the need for a continuous system with a degree of accuracy consistent with the requirements of safe navigation. At present, this requirement is best fulfilled by the Global Positioning System (GPS). The GPS system is referenced to World Geodetic System 1984 (WGS 84) Datum. Using the ellipsoid model for the spherical model attains more accurate calculation of sailing on the Earth. Therefore, we construct a computational procedure for solving the length of the arc of a great ellipse, the waypoints and azimuths along a great ellipse.

In this paper, we provide the straightforward formulae involving the great elliptic sailing based on two scenarios. The first scenario is that the departure point and the destination point are known. The second scenario is that the departure point and the initial azimuth are given.
\end{abstract}

\section{INTRODUCTION}

Since the Earth is flattened slightly at the poles and bulges somewhat at the equator, the geometrical figure used in geodesy to approximate the shape of the Earth is an ellipsoid of revolution obtained by rotating an ellipse about its shorter axis $[1,2]$. An ellipsoid of revolution describing the figure of the Earth is called a reference ellipsoid. Since the Earth is not a perfect sphere, a great circle (GC) becomes a great ellipse (GE). Using the ellipsoid model for the Earth can attain more accurate calculation of the shortest distance between two points on the Earth. The shortest path between two points on the ellipsoid is along the arc of geodesic (Geod).

In traditional navigation, the computations are simplified

Paper submitted 11/05/08; revised 06/12/09; accepted 06/16/09. Author for correspondence: Wei-Kuo Tseng (e-mail: wilco0917@yahoo.com.tw).

*Department of Merchant Marine, National Taiwan Ocean University, Keelung, Taiwan, R.O.C.

**Department of Shipping and Transportation Management, National Taiwan Ocean University, Keelung, Taiwan, R.O.C. by the use of a spherical Earth model. The discrepancies between the results on the spherical and the ellipsoidal mode of the Earth are in order of $0.27 \%$ according to Tobler [6], and in the order of $0.5 \%$ according to Earle [5]. Despite these discrepancies the use of the spherical model in traditional navigation for most practical purpose is considered satifactory. Nevertheless for the case of sailing computations in GIS navigational systems such as ECDIS the computation must be conducted on the ellipsoid in order to eliminate these errors but without seeking the sub meter accuracies pursued in the other geodetic application. Seeking extremely high accuracy for marine navigation purpose dose not offer any real benefit and require more computing power and processing time. For these reasons and before proceeding with the adoption of any geodetic computational method on the ellipsoid for sailing calculation it is required to adopt a realistic accuracy standard in order not only to eliminate the significant errors of the spherical model but also to avoid the exaggerate and unrealistic requirement of sub meter accuracy.

The computation of geodesic needs many iterative procedures. The calculation of great ellipse can reduce the amount of computation and it gives a good approximation to the shortest distance [10]. A lot of specific papers present in detail the advantages and benefits of the Great Ellipse Sailing and Great Circle Sailing $[3,7,8,10,11]$. The work presented here embraces more straightforward derivations of the calculation of a great ellipse based on two scenarios.

The first scenario is that the departure and the destination point are known. The second scenario is that the departure point and the initial azimuth are known. Our approach provides a concise solution of length integral and a simpler azimuth function along a great ellipse that may offer ease of computation in navigation and perhaps can find its virtue in the training environment.

Each position on the surface of the Earth can be considered as a vector drawn from the center to a point on the surface. Using the properties of vector analysis easily gives course function along a great ellipse without applying the rules of spherical trigonometry. Length integral permits an integral expression for the distance between two points. Although the integral for distance of a great ellipse is not mathematic closed form and lacks a convenient anti-derivative, the computation of the integral can nevertheless be carried out efficiently and quickly by numerical integration such as the trapezoidal rules or the Simpson's rule [12]. Many available commercial mathematical software packages can handle definite integrals. 


\section{GEOMETRY OF SPHEROIDAL EARTH}

Since the spherical earth is a convenient assumption, the question of which sphere should be chosen for navigation purposes naturally arises. A sphere having equal volume or surface area could be selected, but neither of these is as convenient as the navigation sphere defined here that one span of one minute of arc on any great circle is equal to one International Nautical mile (n.m) of 1852 meters. The navigation sphere therefore has a radius of $a=60 \times 360 / \pi$ or $3437.7468 \mathrm{~nm}$.

The Earth is a spheroid produced by an ellipsoid of revolution having small eccentricity as currently defined by the World Geodetic Standard of 1984 (WGS84), whose minor axis is the polar axis and major axis lies in the equatorial plane. The Earth's equatorial radius or semi-major radius $(a)$ is the distance from its center to the equator and is approximately equal to $6,378,137 \mathrm{~km}$. A nature unit of distance is the span of one minute of longitude at the equatorial arc also called the geographic mile. It is equal to 1855.3249 meters and 1.0018 nautical miles. For navigation purposes, the semi-major radius is taken to be equal to $60 \times 360 / \pi$ and thus has the value of $a=3437.747$ geographic miles. The Earth's polar radius or semi-minor radius $b$ is the distance from its center to the North and South Poles, and is equal to $6,356.750 \mathrm{~km}$. The eccentricity is approximately equal to $e=0.08181919$ on the WGS84 ellipsoid.

A point on the surface of the Earth can be identified by spherical coordinates with longitude $\lambda$ and latitude $\phi$. Because the Earth is slightly flattened by its rotation, cartographers refer to a variety of auxiliary latitudes to precisely adapt spherical projections according to their different purposes. In common usage, "latitude" refers to geodetic or geographic latitude $\varphi$ and is the angle between the equatorial plane and a line that is normal to the reference spheroid. Reduced or parametric latitude $\beta$ is the angle at a sphere that is tangent to the ellipsoid along the equator. The geocentric latitude $\phi$ is the angle between the plane of equator and a line from the center of the Earth to the point whose geocentric latitude is being defined. A graphical illustration of these latitudes can be found in Fig. 1. Equation (1) presents the relation between geodetic latitude and reduced latitude and (2) states the relation between geocentric latitude and geodetic latitude: (All the symbols used in the following are listed in the appendix for quick reference.)

$$
\begin{aligned}
& \tan \beta=\left(1-e^{2}\right)^{\frac{1}{2}} \tan \varphi \\
& \tan \phi=\left(1-e^{2}\right) \tan \varphi
\end{aligned}
$$

where $e$ is eccentricity of the ellipse.

Using reduced latitude or geographic latitude, a point $P$ on the surface of the Earth can be represented as a function of $\lambda$ and $\beta$.

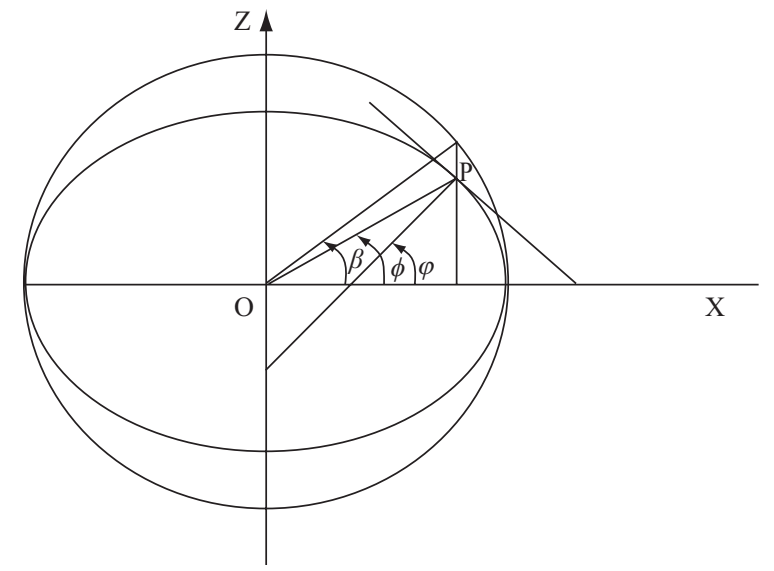

Fig. 1. Geographic latitude, geocentric latitude and reduced latitude.

$$
P(\beta, \lambda)=\left[\begin{array}{l}
a \cos \beta \cos \lambda \\
a \cos \beta \sin \lambda \\
b \sin \beta
\end{array}\right]^{T}
$$

where $a$ and $b$ are the semi-major radius and semi-minor radius of the ellipsoid respectively. Likewise, $P$ also can be represented as a function of $\lambda$ and $\varphi$.

$$
P(\varphi, \lambda)=\left[\begin{array}{l}
N \cos \varphi \cos \lambda \\
N \cos \varphi \sin \lambda \\
N\left(1-e^{2}\right) \sin \varphi
\end{array}\right]^{T}
$$

where $N$ is the radius of curvature of the prime vertical: $N=a /\left(1-e^{2} \sin ^{2} \varphi\right)^{1 / 2}$.

\section{THE DISTANCE ON THE ELLIPSOID}

The definition of the length of a smooth path is as the following:

If $F:\left[t_{1}, t_{2}\right] \rightarrow R^{3}$ is a smooth path with coordinate functions [12]

$$
x=f_{x}(t), y=f_{y}(t), z=f_{z}(t), t \in\left[t_{1}, t_{2}\right],
$$

then its distance is given by

$$
D=\int_{t_{1}}^{t_{2}} \sqrt{\left|f_{x}^{\prime}(t)\right|+\left|f_{y}{ }^{\prime}(t)\right|+\left|f_{z}^{\prime}(t)\right|} d t \text {. }
$$

Differentiating $P$ in (3) with respect to $\lambda$ obtains:

$$
\dot{P}=\left[\begin{array}{l}
-a \sin \beta \cos \lambda \frac{d \beta}{d \lambda}-a \cos \beta \sin \lambda \\
-a \sin \beta \sin \lambda \frac{d \beta}{d \lambda}+a \cos \beta \cos \lambda \\
b \cos \beta \frac{d \beta}{d \lambda}
\end{array}\right]^{T}
$$


By definition, the distance between two points on the surface of ellipsoid of revolution can be determined by (6)

$$
s\left(\lambda_{1}, \lambda_{2}\right)=\int_{\lambda_{1}}^{\lambda_{2}} \sqrt{\left(a^{2} \sin ^{2} \beta+b^{2} \cos ^{2} \beta\right)\left(\frac{d \beta}{d \lambda}\right)^{2}+a^{2} \cos ^{2} \beta} d \lambda
$$

In convention, since navigators present the position on the Earth by the geographic latitude, the expression of reduced latitude in the RHS of (6) must be substituted by the expression of geographic latitude. Using the chain rule for derivatives yields

$$
\frac{d \varphi}{d \lambda}=\frac{d \beta}{d \varphi} \frac{d \varphi}{d \lambda}
$$

Differentiating (1) gives

$$
\frac{d \beta}{d \varphi}=\left(1-e^{2}\right)^{\frac{1}{2}} \frac{\sec ^{2} \varphi}{\sec ^{2} \beta}=\left(1-e^{2}\right)^{\frac{1}{2}} \frac{1+\tan ^{2} \varphi}{1+\left(1-e^{2}\right) \tan ^{2} \varphi}
$$

Then, setting $\gamma=1-e^{2}$ and substituting (6) into Equation obtains

$s\left(\lambda_{1}, \lambda_{2}\right)=a \int_{\lambda_{1}}^{\lambda_{2}} \sqrt{\frac{1}{1+\gamma \tan ^{2} \varphi}\left(1+\gamma^{2}\left(\frac{\left(1+\tan ^{2} \varphi\right)^{3}}{\left(1+\gamma \tan ^{2} \varphi\right)^{2}}\left(\frac{d \varphi}{d \lambda}\right)^{2}\right)\right.} d \lambda$

\section{THE EQUATION OF A GREAT ELLIPSE}

Three unit vectors associated with the vector $P$ are the unit velocity vector $\vec{V}$ which characterizes its moving direction, the meridian tangent vector $\vec{N}$ which points the North Pole and the parallel tangent vector $\vec{E}$ which points to the East. The azimuth $\alpha$ is the angle between the meridian plane and the normal plane at point $P$ containing the velocity vector $\vec{V}$. For convenience, in the subsequent of this paper, a subscript will be added to designate the notation which is associated with a specific point. For example, $\vec{V}_{a}$ denotes the unit velocity vector of point $A$. If no subscript is specified, the notation is associated with an arbitrary waypoint under consideration. The notations of latitudes and longitudes are treated in the same way.

Partial differentiating $P$ in (3) with respect to $\lambda$ and $\varphi$ accordingly and following Fig. 2, Fig. 3 obtain:

$$
\vec{E}=(-\sin \lambda, \cos \lambda, 0)
$$

$$
\vec{N}=(-\sin \varphi \cos \lambda,-\sin \varphi \sin \lambda, \cos \varphi)
$$

Since the two vectors $\vec{E}$ and $\vec{N}$ form a basis for the set of

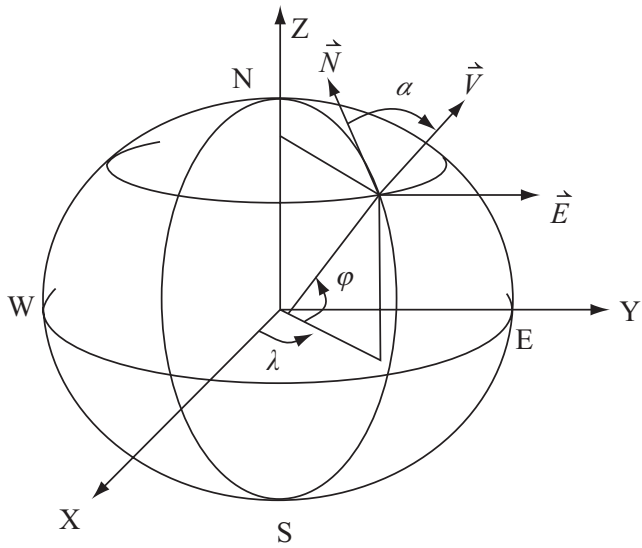

Fig. 2. Geographic latitude, longitude, and azimuth.

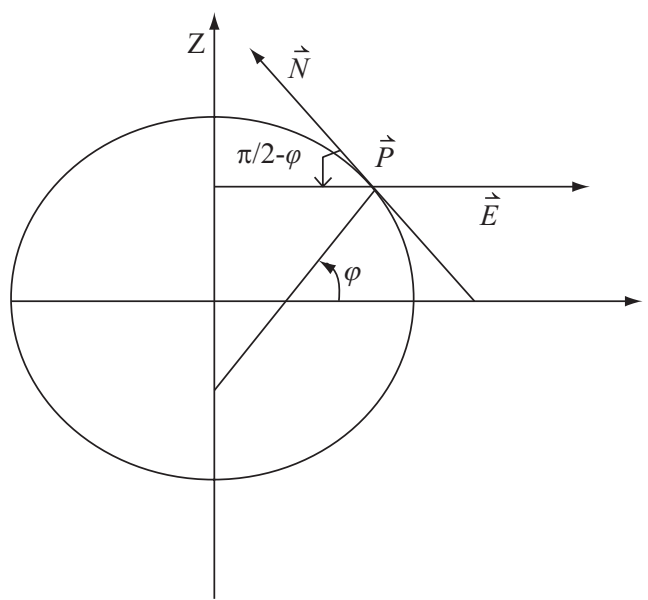

Fig. 3. The north and the east vectors at a point on the ellipsoid.

all vectors in a tangent plane on a spheroid, the velocity vector $\vec{V}$ is a linear combination of $\vec{E}$ and $\vec{N}$ (Fig. 4), which is shown in (12):

$$
\vec{V}=\sin \alpha \cdot \vec{E}+\cos \alpha \cdot \vec{N}
$$

A great ellipse is defined as the intersection of a spheroid and a plane passing its origin $[7,8]$. Two scenarios for determining a great ellipse are considered here:

1. Determining a great ellipse by two points on a spheroid.

2. Determining a great ellipse by a point and its course angle.

\section{Scenario 1: Determining a great ellipse by two points on a spheroid.}

Suppose $\vec{A}$ and $\vec{B}$ are the vectors of the departure and the destination accordingly. The $\vec{P}$ is any waypoint of the great ellipse determined by the vector $\vec{A}$ and the vector $\vec{B}$. By definition of equation of plane, a plane containing the great ellipse can be governed by (13). 


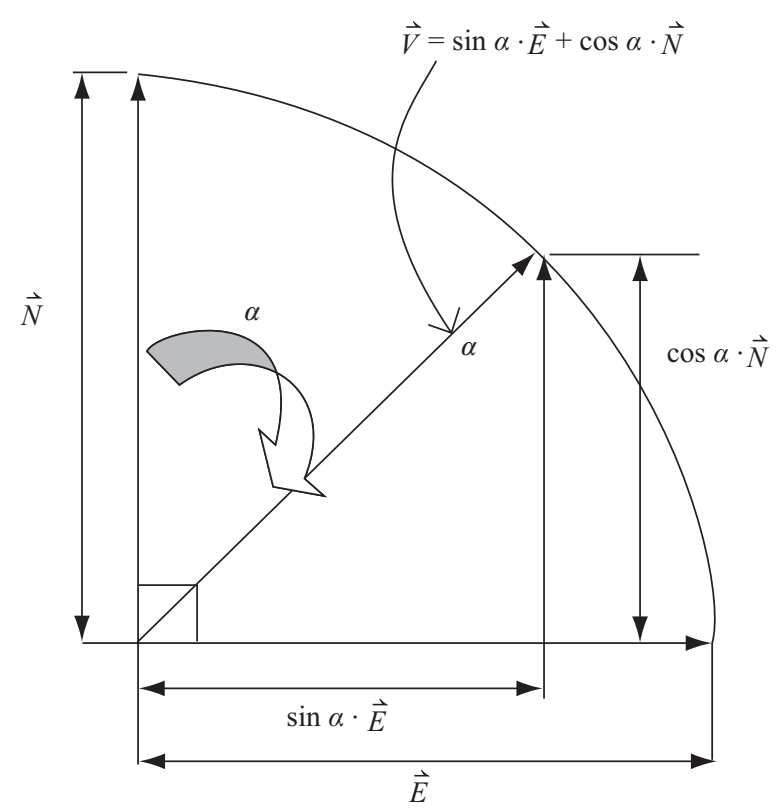

Fig. 4. The linear combination of the north vector and the east vector.

$$
l \cdot x+m \cdot y+1 \cdot z=0
$$

Note that $\vec{n}=(l, m, 1)$ is the normal to the plane. Since $\vec{A}$, $\vec{B}$, and $\vec{P}$ are on the plane of the great ellipse, we have

$$
\left\{\begin{array}{l}
\vec{n} \cdot \vec{A}=0 \\
\vec{n} \cdot \vec{B}=0 \\
\vec{n} \cdot \vec{P}=0
\end{array}\right.
$$

which is equivalent to $\vec{A} \times \vec{B} \cdot \vec{P}=0$, i.e., the their scalar triple product is zero $[4,7,8,12]$.

By solving

$$
\left\{\begin{array}{l}
\vec{n} \cdot \vec{A}=0 \\
\vec{n} \cdot \vec{B}=0
\end{array}\right.
$$

we have

$$
\left\{\left[\begin{array}{l}
l \\
m
\end{array}\right]=-\left[\begin{array}{cc}
\cos \varphi_{a} \cos \lambda_{a} & \cos \varphi_{a} \sin \lambda_{a} \\
\cos \varphi_{b} \cos \lambda_{b} & \cos \varphi_{b} \sin \lambda_{b}
\end{array}\right]^{-1}\left[\begin{array}{c}
\left(1-e^{2}\right) \sin \varphi_{a} \\
\left(1-e^{2}\right) \sin \varphi_{a}
\end{array}\right]\right.
$$

Note that if the departure vector $\vec{A}$ and the destination vector $\vec{B}$ are known, the waypoint $\vec{P}$ along the great ellipse must lie in the plane spanned by $\vec{A}$ and $\vec{B}$. Hence their scalar triple product will be zero.

Expanding (15) yields the following.

$$
\left\{\begin{array}{l}
l=\frac{\cos \varphi_{a} \sin \lambda_{a}\left(1-e^{2}\right) \sin \varphi_{b}-\cos \varphi_{b} \sin \lambda_{b}\left(1-e^{2}\right) \sin \varphi_{a}}{\cos \varphi_{a} \cos \lambda_{a} \cos \varphi_{b} \sin \lambda_{b}-\cos \varphi_{a} \sin \lambda_{a} \cos \varphi_{b} \cos \lambda_{b}} \\
m=\frac{-\cos \varphi_{a} \cos \lambda_{a}\left(1-e^{2}\right) \sin \varphi_{b}+\cos \varphi_{b} \cos \lambda_{a}\left(1-e^{2}\right) \sin \varphi_{a}}{\cos \varphi_{a} \cos \lambda_{a} \cos \varphi_{b} \sin \lambda_{b}-\cos \varphi_{a} \sin \lambda_{a} \cos \varphi_{b} \cos \lambda_{b}}
\end{array}\right.
$$

Since $\vec{n} \cdot \vec{P}=0$, we have

$$
l \cdot \cos \varphi \cos \lambda+m \cdot \cos \varphi \sin \lambda+\left(1-e^{2}\right) \sin \varphi=0
$$

Rearranging and writing (17) as a tangent function, we arrive at

$$
\tan \varphi=-(l \cdot \cos \lambda+m \cdot \sin \lambda) /\left(1-e^{2}\right)
$$

From (18), the geographic latitude of any point on the great ellipse determined by two points can be identified once the longitude is specified. To determine the longitude for given latitude, Eq. (18) can be transformed into (19) by using a trigonometric formula.

$$
\lambda=\sin ^{-1}\left[\frac{\left(1-e^{2}\right)}{\sqrt{l^{2}+m^{2}}} \tan \varphi\right]+\Delta
$$

where $\Delta=\tan ^{-1}\left(\frac{l}{m}\right)$.

Since (9) requires the derivative $d \varphi / d \lambda$, differentiating (18) yields

$$
\frac{d \varphi}{d \lambda}=\frac{(l \cdot \sin \lambda-m \cdot \cos \lambda)}{\left(1-e^{2}\right)\left(1+\tan ^{2} \varphi\right)}
$$

Substituting (20) into (9) yields

$$
\begin{aligned}
& s\left(\lambda_{1}, \lambda_{2}\right) \\
& \quad=a \int_{\lambda_{1}}^{\lambda_{2}} \sqrt{\frac{1}{1+\gamma \tan ^{2} \varphi}\left(1+\frac{1+\tan ^{2} \varphi}{\left(1+\gamma \tan ^{2} \varphi\right)^{2}}(l \sin \lambda-m \cos \lambda)^{2}\right)} d \lambda
\end{aligned}
$$

\section{Scenario 2: Determining a great ellipse by a point and its course angle.}

Let the vector of the given point be $\vec{A}$. Since its course angle $\alpha_{a}$ is known, the velocity vector $\vec{V}_{a}$ can be obtained by linear combination of the meridian tangent vector $\vec{N}_{a}$ and the parallel tangent vector $\vec{E}_{a}$ as the following.

$$
\vec{V}_{a}=\sin \alpha_{a} \cdot \vec{E}_{a}+\cos \alpha_{a} \cdot \vec{N}_{a}
$$


Since both $\vec{A}$ and $\vec{V}_{a}$ lie in the plane of the great ellipse, we have

$$
\left\{\begin{array}{l}
\vec{n} \cdot \vec{A}=0 \\
\vec{n} \cdot \vec{V}_{a}=0
\end{array}\right.
$$

We can solve (23) for $l$ and $m$. Once $l$ and $m$ are determined, the derivation of the equation for the great ellipse is the same as scenario 1 .

\section{THE VERTICES AND NODES OF A GREAT ELLIPSE}

When danger is likely to be encountered in high latitudes, the great ellipse path must be modified to avoid it. For this reason, a navigator may want to know whether the highest (lowest) latitude or the vertex reached by his path is safe and at what longitude it will occur. For example, in case of bad weather, the navigator will choose to sail a part of his track at a parallel circle. A vertex ( $N$ or $S$ vertex), whose latitude and longitude are denoted as $\varphi_{v}$ and $\lambda_{v}$, occurs when $d \varphi / d \lambda=0$. Setting (20) to be zero gives the following.

$$
\frac{d \varphi}{d \lambda}=\frac{l \sin \lambda-m \cos \lambda}{\left(1-e^{2}\right) \sec ^{2} \varphi}=0
$$

we have

$$
\lambda_{v}=\tan ^{-1}\left(\frac{m}{l}\right)+n \pi, n \in(0,1)
$$

Substituting (25) into (18) yields the latitude of the vertex:

$$
\varphi_{v}= \pm \tan ^{-1}\left(\frac{\sqrt{l^{2}+m^{2}}}{1-e^{2}}\right)
$$

Setting the geography latitude $\varphi=0$ in (18) gives the ascending and descending nodes where the great ellipse intersects with the equator at longitude $\lambda_{e}$ as the following:

$$
\lambda_{e}=\tan ^{-1}\left(\frac{l}{m}\right)+n \pi, n \in(0,1)
$$

which is equivalent to $\lambda_{e}=\lambda_{v} \pm \pi / 2$.

Substituting (26) and (27) into (19) yields a different presentation for the equation of a great ellipse as the following:

$$
\sin \left(\lambda-\lambda_{e}\right)=\frac{\tan \varphi}{\tan \varphi_{v}}
$$

which is the same as the equation obtained by Napier's mnemonic Rule for Right-Angle Triangle [1] for conventional technique of navigation.

\section{THE COURSE AT ANY POINT IN A GREAT ELLIPSE}

The velocity vector of any waypoint on a great ellipse satisfies (12). That is,

$$
\begin{aligned}
\vec{V} & =\sin \alpha \cdot \vec{E}+\cos \alpha \cdot \vec{N} \\
& =\left[\begin{array}{c}
-\sin \lambda \sin \alpha-\cos \lambda \sin \varphi \cos \alpha \\
\cos \lambda \sin \alpha-\sin \lambda \sin \varphi \cos \alpha \\
\cos \varphi \cos \alpha
\end{array}\right]^{T}
\end{aligned}
$$

Since the vector $\vec{V}$ lies in the plane of a great ellipse, then the dot product of the vector $\vec{V}$ and the normal vector of the great ellipse equals to zero.

$$
\vec{n} \cdot \vec{V}=0
$$

Expanding (30) gives

$\sin \alpha(-l \sin \lambda+m \cos \lambda)-\cos \alpha$

$$
(l \cos \lambda \sin \varphi+m \sin \lambda \sin \varphi+\cos \varphi)=0
$$

Rearranging (31) and incorporating (18), we have

$$
\tan \alpha=\cos \varphi \frac{1+\left(1-e^{2}\right) \tan ^{2} \varphi}{(l \sin \lambda-m \cos \lambda)}
$$

When eccentricity $e=0$, Eq. (32) can be reduced into (33):

$$
\tan \alpha=\frac{\sec \varphi}{l \sin \lambda-m \cos \lambda}
$$

Therefore, the azimuth of any waypoint on a great ellipse is

$$
\alpha=\tan ^{-1}\left(\cos \varphi \frac{1+\left(1-e^{2}\right) \tan ^{2} \varphi}{(l \sin \lambda-m \cos \lambda)}\right)
$$

and the azimuth of any waypoint on a great circle is

$$
\alpha=\tan ^{-1}\left(\frac{\sec \varphi}{l \sin \lambda-m \cos \lambda}\right)
$$

The formulation of the azimuth of any waypoint on a great ellipse in [4] is expressed as the 


$$
\alpha=\cos ^{-1}\left[\frac{\left(\vec{P} \times V_{p}\right) \cdot(\vec{P} \times \vec{B})}{\left|\vec{P} \times V_{p} \| \vec{P} \times \vec{B}\right|}\right]
$$

where $V_{p}=(0,0,1)$ is the unit polar vector from the origin to the North Pole. One of the problems with (36) is that course angle at $P_{b}$ can not be resolved with Eq. (36). To deal with this problem, Eq. (36) can be reformulated as:

$$
\alpha=\cos ^{-1}\left[\frac{\left(\vec{P} \times V_{p}\right) \cdot(\vec{A} \times \vec{B})}{\left|\vec{P} \times V_{p} \| \vec{A} \times \vec{B}\right|}\right]
$$

This equation avoids a discontinuity that can occur as $P$ meets $B$ and is there more versatile.

However, another problem with (36) and (37) arises that $\vec{P} \times \vec{B}$ and $\vec{A} \times \vec{B}$ is the normal to the plane containing a great ellipse. That is, the azimuth measured calculated in (36) or (37) is the angle between the meridian plane passing point $P$ and the plane of the great ellipse. This is incorrect! As we noted in Section IV, the azimuth is the angle between the meridian plan and the normal plane containing the velocity vector $\vec{V}$ of the point $P$. To tackle this problem, we further reformulate (38) as

$$
\alpha=\operatorname{arcos}\left[\frac{\left(\vec{P} \times V_{p}\right) \cdot(\vec{E} \times \vec{N} \times \vec{V})}{\left|\vec{P} \times V_{p} \| \vec{E} \times \vec{N} \times \vec{V}\right|}\right]
$$

In (38), the triple product $\vec{E} \times \vec{N} \times \vec{V}$ is the normal to the normal plane containing the velocity vector $\vec{V}$.

For comparison, the computation results for a great ellipse, a great circle, a geodesics, and Earle's formulation departed from $A$ point (Lat $35 \mathrm{~N}$ long $121 \mathrm{E}$ ) to $B$ point (Lat $46.2 \mathrm{~N}$ Long $144 \mathrm{~W}$ ) are shown in Tables 1 and 2. Using very accurate methods for the calculation of long geodesics, as the method of Vicenty (1975) [9] spending a lot of processing time, gives the distance and azimuth on WGS-84 ellipsoid as shown in the following Tables. The result of calculation of geodesics is our benchmark to compare the methods of GC and GE with the method of Geodesics.

The differences of azimuths between GC and Geodesic are significantly smaller than the differences of azimuths between $\mathrm{GC}$ and Geodesics. In this example, the difference in calculated distances on the spherical model (great circle sailing) can exceed $23 \mathrm{~km}$ (about 13 nautical miles). The discrepancies between the distances of the great ellipse and the geodesics are negligible.

We found that the method of GE eliminates the significant errors of the spherical model and avoids the exaggerated and unrealistic requirement of sub meter accuracy. The great ellipse is to be used for accurate sailing calculations on the ellipsoid in a GIS environment. Their validity in terms of the accuracy achieved is assessed and compared to geodesics method.
Table 1. Azimuth Comparison of a great ellipse, great circle, and Geodesic.

\begin{tabular}{rcccccc}
\hline Long & Lat & GC & GE & Geodesic & $\begin{array}{c}\text { Earle's } \\
\text { result }\end{array}$ & Errror \\
\hline 121 & 35.000 & 47.772 & 47.900 & 47.889 & 47.900 & -0.000142 \\
\hline 130 & 40.857 & 53.318 & 53.424 & 53.412 & 53.424 & -0.000151 \\
\hline 140 & 45.646 & 60.185 & 60.266 & 60.255 & 60.267 & -0.000139 \\
\hline 150 & 48.981 & 67.548 & 67.606 & 67.596 & 67.606 & -0.000112 \\
\hline 160 & 51.149 & 75.225 & 75.262 & 75.252 & 75.262 & -0.000076 \\
\hline 170 & 52.338 & 83.085 & 83.103 & 83.093 & 83.103 & -0.000036 \\
\hline 180 & 52.652 & 91.026 & 91.023 & 91.013 & 91.023 & 0.000005 \\
\hline 190 & 52.118 & 98.955 & 98.932 & 98.922 & 98.932 & 0.000047 \\
\hline 200 & 50.689 & 106.779 & 106.736 & 106.725 & 106.736 & 0.000086 \\
\hline 210 & 48.242 & 114.389 & 114.324 & 114.317 & 114.324 & 0.000120 \\
\hline 216 & 46.200 & 118.795 & 118.717 & 118.724 & NA & NA \\
\hline & & & & & & \\
\hline
\end{tabular}

Table 2. Distance Comparison of a great ellipse, a great circle, and a geodesic.

\begin{tabular}{rrrrrrr}
\hline Long & Lat & \multicolumn{1}{c}{ GC } & \multicolumn{1}{c}{ GE } & \multicolumn{1}{c}{ Geod } & GC-Geod(m) & GE-Geod(m) \\
\hline 121 & 35.000 & 0.000 & 0.000 & 0.000 & 0.000 & 0.000 \\
\hline 130 & 40.857 & 551.673 & 552.427 & 552.427 & -1396.712 & 0.007 \\
\hline 140 & 45.646 & 1074.065 & 1076.017 & 1076.017 & -3614.460 & 0.014 \\
\hline 150 & 48.981 & 1526.932 & 1530.245 & 1530.245 & -6136.159 & 0.020 \\
\hline 160 & 51.149 & 1933.063 & 1937.792 & 1937.792 & -8759.188 & 0.024 \\
\hline 170 & 52.338 & 2311.040 & 2317.198 & 2317.198 & -11403.177 & 0.028 \\
\hline 180 & 52.652 & 2676.530 & 2684.115 & 2684.115 & -14046.802 & 0.031 \\
\hline 190 & 52.118 & 3043.846 & 3052.858 & 3052.858 & -16690.851 & 0.034 \\
\hline 200 & 50.689 & 3427.499 & 3437.938 & 3437.938 & -19333.043 & 0.038 \\
\hline 210 & 48.242 & 3843.760 & 3855.607 & 3855.607 & -21941.152 & 0.043 \\
\hline 216 & 46.200 & 4117.133 & 4129.796 & 4129.796 & -23451.092 & 0.044 \\
\hline
\end{tabular}

\section{CONCLUSION}

In this paper, we have presented an accurate method for computing the distance, position and azimuth along a great ellipse. We also give a different scenario to develop the great ellipse equation determined by a point and its course angle. The method of scenario 2 especially is suitable for calculation of area of missile attack. With basic vector analysis, the derivation presented in this paper is more straightforward and concise than the formulation developed in [3]. We have developed a correct course function as a substitute of the course function developed in [3], which mistreated the plane of a great ellipse as a normal plane. The great ellipse is to be used for accurate sailing calculations on the ellipsoid in a GIS environment. Their validity and effectiveness in terms of the accuracy achieved and the calculation time required are assessed and compared to standard geodetic method. The results of our formulation can be easily implemented as software modules such as Add-in of Excel to facilitate navigators to plan their routes of sail more accurately. The approach was intended to appeal to the navigator who are interested in the 
mathematics of navigation and who, nowadays, solves his problems of navigation with a personal computer.

\section{APPENDIX}

Symbol Glossary

$\begin{array}{ll}a & \text { semi-major radius } \\ b & \text { semi-minor radius } \\ e & \text { eccentricity (WGS84) } \\ \lambda & \text { longitude } \\ \varphi & \text { geographic latitude } \\ \beta & \text { Reduced or parametric latitude } \\ \phi & \text { geocentric latitude } \\ \vec{N} & \text { meridian tangent vector } \\ \vec{E} & \text { parallel tangent vector } \\ \vec{V} & \text { unit velocity vector } \\ \vec{n}=(l, m, 1) & \text { Normal to plane of great ellipse } \\ \lambda_{v} & \text { longitude of vertex } \\ \varphi_{v} & \text { latitude of vertex } \\ \lambda_{e} & \text { longitude of node }\end{array}$

\section{REFERENCES}

1. Bowditch, N., The American Practical Navigator, Vol. II, Defense Mapping Agency (1981).

2. Chou, H., Geo Navigation, Maritime Research Center, National Taiwan Ocean University, Taiwan, (1999). (In Chinese)

3. Earle, M. A., "A vector solution for navigation on a great ellipse," The Journal of Navigation, Vol. 53, pp. 473-481 (2000).

4. Earle, M. A., "A vector solution for great circle navigation," The Journal of navigation, Vol. 58, pp. 451-457 (2005).

5. Earle, M. A., "Sphere to spheroid comparison," The Journal of navigation, Vol. 59, pp. 491-496 (2006).

6. Tobler, W. R., "A comparison of spherical and ellipsoidal measures," The Professional Geographer, Vol. XVI, No. 4, pp. 9-12 (1964).

7. Tseng, W. K. and Lee, H. S., "The vector function of traveling distance for great circle navigation," The Journal of Navigation, Vol. 60, pp. 158-164 (2007).

8. Tseng, W. K. and Lee, H. S., "Building the latitude equation of the midlongitude," The Journal of Navigation, Vol. 60, pp. 164-170 (2007).

9. Vincenty, T., "Direct and inverse solutions of geodesics on the ellipsoid with application of nested equations," Survey Review, Vol. XXII, No. 176, p.p. 88-93 (1975).

10. Walwyn, P. R., "The great ellipse solution for distances and headings to steer between waypoints," The Journal of Navigation, Vol. 52, pp. 421-424 (1990).

11. Williams, R., Geometry of Navigation, Horwood Publishing, Chichester UK ISBN 1-898563-46-2 (1998).

12. Wylie, C. Ray and Barrett, Louis C., Advanced Engineering Mathematics, McGraw-Hill, pp. 834 (1982). 